Publ. RIMS, Kyoto Univ.

15 (1979), 279-287

\title{
On Hironaka's Monoideal
}

\author{
By \\ Tohsuke URABE*
}

\section{§o. Introduction}

The aim of this article is to verify a fundamental property of what we call "generic monoideal" associated with a defining ideal of an algebraic scheme.

To be precise, let $X$ be an algebraic subscheme of a regular algebraic scheme $Z$. Let $\eta$ be a point of $X$, let $L$ be the local ring of $Z$ and let $K$ be the residue field of $L$, i.e., $K=L / M$ with the maximal ideal $M$ of $L$. We denote by $I$ the ideal of the tangent cone of $X$ at $\eta$ in the graded algebra $g r_{M}(L)$. If we pick a regular system of parameters of $L$, we obtain a presentation

$$
g r_{M}(L)=K\left[\approx_{1}, \cdots, z_{n}\right]
$$

with the induced parameters $\approx=\left(\approx_{1}, \cdots, \approx_{n}\right)$ (indeterminates over $K$ where $n$ is the dimension of $L$ ). The ideal $I$ in $K[z]$ is then homogeneous. We extract certain exponents (by means of the lexicographical order in $Z_{0}^{n}$ ) from the polynomial expressions of elements of $I$. (cf. Notation and definition 1.1.) Those exponents form a monoideal in the additive semi-group $Z_{0}^{n}$, denoted by $E(z ; I)$. For almost all (or non-special) choice of $z$, we obtain $E(I)=E(z ; I)$ independent of $\approx$. This $E(I)$ is what we call the generic monoideal of $I$. Hironaka (Remark 5.4 of [1], p. 79) claimed without proof that this $E(I)$ is monotonous. Assuming this, he verified the existence of the local distinguished presentation ([1], p. 83), which plays an important role in the resolution of singularities of "algebraic spaces" in the sense of Artin and Knudson.

The theorem (Th. 2.2) we prove in this article is that the generic $E(I)$ has a much stronger property than just being monotonous. As a

Communicated by H. Hironaka, September 2, 1978.

* Research Institute for Mathematical Sciences, Kyoto University. 
matter of fact, by examining the stronger property ("strongly monotonous", Def. 2.1) we see clearly how the characteristic of $K$ affects such a property. From our theorem, we can deduce not only what Hironaka wanted but also a complete (and simple) characterization of $E(I)$ in the case of char $=0$ and $n=2$, just as expected by Horikawa. We do not yet have any complete (and hopefully simple) characterization of those monoideals that are realized as $E(I)$. Clearly $E(I)$ has more complicated structure in the case of $n \geqq 3$ and hence should represent some interesting invariants of the ideal $I$.

\section{Supplement to Introduction, by Heisuke Hironaka}

I would like to add to the introduction, some comments that might help the readers to better understand the motivation of Mr. Urabe's work. Recently I found that my proof of the monotonous property of $E(I)$ was incomplete and not easy to modify for completion. Thus the claim of Remark 5. 4 of [1] has been left unproved until this work of Mr. Urabe. This contains some ingenious calculative technique which is interesting by itself and should be useful for further characterization of the structure of $E(I)$ in general.

\section{$\S 1$. Preliminaries}

Notation and definition 1. 1. We fix a field $K$ with the characteistic $\operatorname{ch} K=p$, let $Z_{0}$ denote the additive semi-group of non-negative integers, and let $z$ always denote $n$ variables $z=\left(z_{1}, \cdots, z_{n}\right)$, and for $A$ $=\left(a_{1}, \cdots, a_{n}\right) \in Z_{0}^{n}, z^{4}=\prod_{i=1}^{n} z_{i}^{a_{i}}$.

In the set $Z_{0}^{n}$ the lexicographical order $>$ is defined as follows:

$$
\begin{aligned}
& A=\left(a_{1}, \cdots, a_{n}\right)>B=\left(b_{1}, \cdots, b_{n}\right) \\
\Leftrightarrow & \text { there exists an integer } k \text { such that } a_{i}=b_{i} \text { for } i<k, \\
& \text { and } a_{k}>b_{k}, \text { where } 1 \leqq k \leqq n .
\end{aligned}
$$

Every non-zero element $F \in K[z]$ can be written as $F=\sum_{A \in Z_{0}^{n}} F_{A} z^{A}$ with $F_{A} \in K$, where $F_{A}=0$ for all but a finite number of $A$ 's. We define

$$
\operatorname{lex}_{z}(F)=\max \left\{A \in Z_{0}^{n} \mid F_{A} \neq 0\right\}
$$


where max means the maximum element in the lexicographical order.

We say a subset $E$ in $Z_{0}^{n}$ is a monoideal if

$$
E+Z_{0}^{n}=E
$$

Now for a homogeneous jdeal $I$ in $K[\approx]$ we define

$$
E(\approx ; I)=\left\{\operatorname{lex}_{z}(F) \mid 0 \neq F \in I\right\}
$$

which is a monoideal in $Z_{0}^{n}$ and we shall sometimes denote it simply by $E(\approx)$.

This $E(z ; I)$ depends on the coordinate system but we can define a similar notion which is independent of the choice of the coordinate system.

Let $R$ be a domain containing $K$ such that $R$ and $K[z]$ are algebraically independent over $K$, and let $u=\left(u_{i j}\right)$ be an $n \times n$-matrix with entries in $R$ and with $\operatorname{det}\left(u_{i j}\right) \neq 0$. We define

$$
\tau=\left(w_{1}, \cdots, w_{n}\right)=z^{u} \Leftrightarrow z_{i}=\sum_{i=1}^{n} u_{i j} w_{j}, \quad 1 \leqq i \leqq n .
$$

we call easily check $z^{u u^{\prime}}=\left(z^{u}\right)^{u^{\prime}}$. Then for $w^{\prime}=z^{u}, w_{1}, \cdots, w_{n}$ are indeterminates over $R$ and we can regard $R[w]$ as a $K[z]$-algebra. When $u$ is invertible, we note that $R[r]=R[z]$.

Lemma 1.2. If $u_{i j}(1 \leqq i, j \leqq n)$ are algebraically independent over $K[\approx]$, then for every $\sigma, \tau \in G L(n, K)$

$$
E\left(\approx^{u} ; \operatorname{IR}\left[z^{u}\right]\right)=E\left(z^{\sigma u \tau} ; \operatorname{IR}\left[z^{\sigma u \tau}\right]\right) .
$$

Proof. Let $R^{\prime}$ be a domain containing $R$ such that $R^{\prime}$ and $K[z]$ are also algebraically independent over $K$, and let $Q, Q^{\prime}$ be respectively the quotient field of $R, R^{\prime}$. Then it is easy to see that

$$
E\left(z^{u} ; \operatorname{IR}\left[z^{u}\right]\right)=E\left(\approx^{u} ; \operatorname{IQ}\left[z^{u}\right]\right)=E\left(z^{u} ; I Q^{\prime}\left[z^{u}\right]\right)=E\left(\approx^{u} ; I R^{\prime}\left[z^{u}\right]\right) .
$$

Therefore we may assume $R=K(u)$.

We set

$$
u^{\prime}=\sigma u \tau
$$

$$
w=z^{u}
$$




$$
w^{\prime}=z^{u^{\prime}}
$$

Then there exists a $K$-algebra isomorphism from $K[u]$ to $K\left[u^{\prime}\right]$ which maps $u$ to $u^{\prime}$. This isomorphism extends to an automorphism of $R$ over $K$ which maps $u$ to $u^{\prime}$ and moreover to an automorphism of $R[\approx]$ which fixes every $z_{j}$. By $(1.2 .2)$ and (1.2.3) this isomorphism maps $w_{j}$ to $w_{j}^{\prime}$ for every $j$. We can consequently have an isomorphism $\Phi$, from $R[w]$ to $R\left[w^{\prime}\right]$ over $K[z]$ which maps $w$ to $w^{\prime}$. Of course, $F \in I R[w]$ if and only if $F^{\Phi} \in I R\left[w^{\prime}\right]$ and $\operatorname{lex}_{w}(F)=\operatorname{lex}_{w}\left(F^{\Phi}\right)$, which deduces the desired result.

q.e.d.

This Lemma 1.2 and the above proof show that $E\left(\approx^{u} ; \operatorname{IR}\left[\approx^{u}\right]\right)$ is independent of not only the choice of $z$, the generators of $K[z]$, but also the domain $R$ which contains $u$, and enable us to define

$$
E(I)=E\left(z^{u} ; \operatorname{IR}\left[z^{u}\right]\right)
$$

if $u_{i j}(1 \leqq i, j \leqq n)$ are algebraically independent over $K[z]$. Following the lecture by $\mathrm{H}$. Hironaka at Kyoto University, we call this $E(I)$ the generic monoideal associated with the homogeneous ideal $I$ in $K[z]$.

Remark 1.3. The aim of this article is to prove a characteristic property of this $E(I)$ and to complete the proof of the existence of the local distinguished presentation by Hironaka in [1].

\section{§ 2. A Characteristic Property of $E(I)$}

The objective of this section is to prove the following Theorem 2.2 which, in particular, implies that $E(I)$ is monotonous in the sense of Hironaka. Although this monotonous property was claimed (without any proof) in Remark 5.4 of [1], it was later pointed out by Hironaka himself that his original proof was incomplete and no complete proof was yet found. Thus this work justifies Hironaka's claim and fills the gap in [1].

Definition 2. 1. We say monoideal $E$ in $Z_{0}^{n}$ is strongly monotonous with respect to $p$ for a non-negative integer $p$, if $E$ has the following property: 
if $A=\left(a_{1}, \cdots, a_{r}, \cdots, a_{s}, \cdots, a_{n}\right) \in E, 1 \leqq r<s \leqq n$, and $\left(\begin{array}{l}a_{s} \\ h\end{array}\right) \neq 0(\bmod p)$ for an integer $0 \leqq h \leqq a_{s}$, then $\mathrm{A}^{\prime}=\left(a_{1}^{\prime}, \cdots, a_{r}^{\prime}, \cdots, a_{s}^{\prime}, \cdots, a_{n}^{\prime}\right)=\left(a_{1}, \cdots, a_{r}+h, \cdots, a_{s}-h, \cdots, a_{n}\right) \in E$. Here $a_{i}^{\prime}=a_{i}(i \neq r, s), a_{r}^{\prime}=a_{r}+h, a_{s}^{\prime}=a_{s}-h$ and () denotes the binnmial coefficient.

Hironaka defines $E$ to be monotonous if the same property holds for $s=r+1$ and $h=a_{s}$. Obviously, a strongly monotonous $E$ is monotonous.

Theorem 2.2. For every homogencous ideal $I$ in $K[z]=K\left[z_{1}\right.$, $\left.\cdots, z_{n}\right]$, the generic monoideal $E(I)$ is strongly monotonous with respect to $\operatorname{ch} K$.

The proof is devided into several lemmas. We first set some special notations to be used in the proof.

\section{Notation 2. 3.}

$u_{i j}, 1 \leqq i, j \leqq n, \mathfrak{w}_{j}, 1 \leqq j \leqq n$ : indeterminates over $K$ and let $\approx_{i}=\sum_{j=1}^{n} u_{i j} w_{j}$, $1 \leqq i \leqq n$.

$\Xi=\left(\xi_{i j}\right), \Xi^{\prime}=\left(\xi_{i j}^{\prime}\right): n \times n$-matrices with entries in $Z_{0}$.

$A=\left(a_{1}, \cdots, a_{n}\right), A^{\prime}=\left(a_{1}^{\prime}, \cdots, a_{n}^{\prime}\right), B=\left(b_{1}, \cdots, b_{n}\right), \cdots$ etc.: elements of $Z_{0}^{n}$. $E_{i j}$ : the $(i, j)$-matrix unit, i.e., $E_{i j}=\left(e_{a \beta}\right)$ with $e_{a \beta}=1$ for $(\alpha, \beta)=(i, j)$ and $e_{\alpha \beta \beta}=0$ for the other $(\alpha, \beta)$.

$E_{i}=(0, \cdots, 0,1,0, \cdots, 0)$ : the $i$-th unit vector.

$\Xi !=\prod_{i=1}^{n} \prod_{j=1}^{n} \xi_{i j} !, \quad A !=\prod_{i=1}^{n} a_{i} !$

$$
|A|=\sum_{i=1}^{n} a_{i}
$$$$
\overline{\underline{E}}=\left(\sum_{i=1}^{n} \xi_{i 1}, \sum_{i=1}^{n} \xi_{i 2}, \cdots, \sum_{i=1}^{n} \xi_{i n}\right)
$$$$
|\boldsymbol{E}|=\left(\sum_{j=1}^{n} \xi_{1 j}, \sum_{j=1}^{n} \xi_{2 j}, \cdots, \sum_{j=1}^{n} \xi_{n j}\right)
$$$$
|\underline{\Xi}|=\sum_{i, j=1}^{n} \xi_{i j}
$$

$z^{A}=\prod_{i=1}^{n} z_{i}^{u_{i}}, \quad w^{B}=\prod_{j=1}^{n} w_{j}^{b_{j}}, \quad u^{\Xi}=\prod_{i=1}^{n} \prod_{j=1}^{n} u_{i j}^{\xi_{i j}}$ 
$D_{r s}=\sum_{i=1}^{n} u_{i s} \frac{\partial}{\partial u_{i r}}, 1 \leqq r, s \leqq n:$ a differentiation on $K[u]$

$D_{r s}^{(h)}$ : a higher differentiation formally defined for a non-negative integer $h$ by

$$
D_{r s}^{(h)}=\frac{1}{h !} D_{r s}^{h}
$$

To be precise, for $\phi u_{1 r}^{b_{1}} \cdots u_{n r}^{b_{n}}$ where $\phi$ does not contain the variables $u_{1 r}, \cdots$, $u_{n r}$,

$$
D_{r s}^{(h)}\left(\phi u_{1 r}^{b_{1}} \cdots u_{n r}^{b_{n}}\right)=\sum_{a_{1}+\cdots+a_{n}=h} \phi \cdot \prod_{i=1}^{n}\left(\begin{array}{c}
b_{i} \\
a_{i}
\end{array}\right) u_{i s}^{a_{i}} \cdot u_{i r}^{b_{i}-a_{2}} .
$$

This satisfies the Leibniz formula

$$
D_{r s}^{(h)}(\phi \psi)=\sum_{k=0}^{h} D_{r s}^{(k)}(\phi) D_{r s}^{(h-k)}(\psi)
$$

Lemma 2.4. For every homogeneous polynomial $F \in K[z]$ of degree $m$,

$$
F=\sum_{|A|=m} c_{A} z^{1} \quad \text { with } c_{A} \in K
$$

if we expand it as an element of $K[u][w]$,

$$
F=\sum_{\mid A_{i}=m} F_{A} w^{A} \text { with } F_{A} \in K[u]
$$

then,

$$
F_{A}=\sum_{\bar{\Xi}=A} c_{\cdot \varepsilon \mid} \frac{|\Xi| !}{\Xi !} u^{\varepsilon}
$$

\section{Proof.}

$$
\begin{aligned}
& F=\sum_{|A|=m} c_{A}\left(u_{11} w_{1}+u_{12} w_{2}+\cdots+u_{1 n} w_{n}\right)^{a_{1}} \cdots\left(u_{n 1} w_{1}+\cdots+u_{n n} w_{n}\right)^{a_{n}} \\
& =\sum_{|A|=m} \sum_{\xi_{11}+\cdots+\xi_{1 n}=a_{1}} \cdots \sum_{\xi_{i_{1}}+\cdots+\xi_{n n}=a_{n}} c_{A} \frac{a_{1} !}{\xi_{11} ! \cdots \xi_{1 n} !} \cdots \frac{a_{n} !}{\xi_{n 1} ! \cdots \xi_{n n} !} u_{11}^{\hat{s}_{1}^{1} \cdots u_{n n}^{\hat{\xi}_{n}}} \\
& \times \boldsymbol{w}_{1}^{\hat{s}_{11}+\cdots+\hat{\xi}_{n 1}} \cdots w_{n}^{\hat{\xi}_{1 n}+\cdots+\hat{\xi}_{n n}} \\
& =\sum_{\underline{\xi}=m} c_{|z|} \frac{|\boldsymbol{\Xi}| !}{\boldsymbol{\Xi} !} u^{\varepsilon^{z}} \mathrm{w}^{\bar{\varepsilon}}
\end{aligned}
$$


$=\sum_{|A|=m} \sum_{\bar{\varepsilon}=A} c_{|\Xi|} \frac{|\Xi| !}{\Xi !} u^{\Xi} w^{A}$

q.e.d.

In addition to these $F_{A}$ for $A \in Z_{0}^{n}$, we define $F_{A}=0$ for $A \in Z^{n} \backslash Z_{0}^{n}$. Then,

\section{Lemma 2. 5 .}

$$
D_{r s}\left(F_{A}\right)=a_{s}^{\prime} F_{A^{\prime}} \text {, where } A^{\prime}=A-E_{r}+E_{s}
$$

Moreover

$$
D_{r s}^{(h)}\left(F_{A}\right)=\left(\begin{array}{l}
a_{s}^{\prime} \\
h
\end{array}\right) F_{A^{\prime}} \text { where } A^{\prime}=A-h E_{r}+h E_{s}
$$

Proof. It is enough to prove the first formula. The second formula is easily deduced from the first one by induction on $h$ in view of the fact that $K$ can be realized as homomorphic image of a domain of the characteristic 0 .

$$
\begin{aligned}
& D_{r s}\left(F_{A}\right)
\end{aligned}
$$

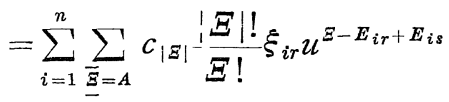

$$
\begin{aligned}
& =\sum_{i=1}^{n} \sum_{\underline{\Xi}=A, \xi_{i r}>0} c_{|\Xi|} \frac{|\boldsymbol{E}| !}{\Xi !} \xi_{i r} u^{\bar{E}-E_{i r}+E_{i s}} \\
& =\sum_{i=1}^{n} \sum_{\overline{\Xi^{\prime}}=A^{\prime}, \xi_{i s^{\prime}}>0} c_{\left|\Xi^{\prime}\right|} \frac{\left|\Xi^{\prime}\right| !}{\Xi^{\prime} !} \xi_{i s}^{\prime} u^{\Xi^{\prime}} \quad \text { (Here } \Xi^{\prime}=\Xi-E_{i r}+E_{i s} \\
& \text { Note that }|\Xi|=\left|\Xi^{\prime}\right| \text {, and } \frac{\Xi !}{\xi_{i r}}=\frac{\Xi^{\prime} !}{\xi_{i s}^{\prime}} \text {.) } \\
& =\sum_{i=1}^{n} \sum_{\underline{\Xi}=A^{\prime}} c_{|\Xi|} \frac{|\Xi| !}{\Xi !} \xi_{i s} \imath l^{\Xi} \\
& =a_{s}^{\prime} F_{A^{\prime}}
\end{aligned}
$$

Next lemma is a criterion for $A \in Z_{0}^{n}$ in order to belong to the generic monoideal $E(I)$. We decompose $I$ into homogeneous parts, say $I=\oplus_{m=0}^{\infty} I_{m}$. We fix an integer $m \geqq 0$ and pick a basis of $I_{m}$ over $K$, say 
$F_{1}, \cdots, F_{q}$, where $q=\operatorname{dim}_{K} I_{m}$. Let $F=\left(F_{1}, \cdots, F_{q}\right)$. Then we can regard the equalities $(2.4 .1),(2.4 .2),(2.4 .3),(2.5 .1)$ and $(2.5 .2)$ as the equalities among vectors $F=\left(F_{1}, \cdots, F_{q}\right), \quad F_{A}=\left(F_{1, A}, \cdots, F_{q, A}\right)$, and $c_{A}$ $=\left(c_{1, A}, \cdots, c_{q, A}\right)$.

Lemma 2.6. The following tro conditions are equivalent for $A$ $\in Z_{0}^{n}$ with $|A|=m$.

(1) $A \notin E(I)$.

(2) There exists $H_{B} \in K[u]$ for every $B \in Z_{0}^{n}$ with $|B|=m$ and $B \geqq A$ such that

$$
H_{A} F_{A}=\sum_{|B|=m, B>A} H_{B} F_{B} \text { and } H_{A} \neq 0
$$

Proof. Set $R=K[u]$ and $I R_{\Delta}[w]=\oplus_{m=0}^{\infty} I_{m} \cdot R_{\Delta}$ with $J=\operatorname{det}\left(u_{i j}\right)$. Therefore $E(I)_{m}=\{B \in E(I)|| B \mid=m\}=\left\{\operatorname{lex}_{w}(G) \mid G \in I_{m} \cdot R\right\}$

$$
I_{m} \cdot R=\left\{F \cdot{ }^{t} H=\sum_{|B|=m}\left(F_{B} \cdot{ }^{t} H\right) w w^{B} \mid H=\left(H_{1}, \cdots, H_{q}\right) \in R^{q}\right\} .
$$

Hence (1) is equivalent to the condition that if $F_{B} \cdot{ }^{t} H=0$ for every $B \in Z_{0}^{n}$ with $|B|=m$ and $B>A$, then $F_{A} \cdot{ }^{t} H=0$. We know by elementary algebra that this condition is equivalent to (2). Here ${ }^{t} H$ denotes the transpose of the vector $H$. q.e.d.

Now the preparation is completed and we can proceed to prove the main theorem.

Proof of Theorem 2.2. Assume $A \notin E(I), m=|A|$. Let $B_{k}=B$ $-k E_{r}+k E_{s}$ with $1 \leqq r<s \leqq n$ for every $B \in Z_{0}^{n}$. It suffices to prove that if $\left(\begin{array}{c}a_{s}+h \\ h\end{array}\right) \not \equiv 0(\bmod p)$ with $p=c h K$ and $A=\left(a_{1}, \cdots, a_{s}, \cdots, a_{n}\right)$, then $A_{h} \notin E(I)$.

By assumption and by Lemma 2.6, there exists $H_{B} \in K[u]$ for every $B \in Z_{0}^{n}$ and

$$
H_{A} F_{A}=\sum_{|B|=m, B>A} H_{B} F_{B}, \quad H_{A} \neq 0 .
$$

After operating $D^{(h)}=D_{r s}^{(h)}$ on both sides we get an equality

$$
\sum_{k=0}^{h} D^{(h-k)}\left(H_{A}\right) \cdot\left(\begin{array}{c}
a_{s}+k \\
k
\end{array}\right) \cdot F_{A_{k}}
$$




$$
=\sum_{k=0}^{h} \sum_{|\boldsymbol{B}|=n, \boldsymbol{B}>A} D^{(n-k)}\left(H_{B}\right) \cdot\left(\begin{array}{c}
b_{s}+k \\
k
\end{array}\right) \cdot F_{B_{k}}
$$

by Lemma 2.5. Since $B_{k}>A_{k} \geqq A_{h}$ if $B>A$ when $0 \leqq k \leqq h$, and since, moreover,

$$
D^{(0)}\left(H_{A}\right) \cdot\left(\begin{array}{c}
a_{s}+h \\
h
\end{array}\right)=H_{A} \cdot\left(\begin{array}{c}
a_{s}+h \\
h
\end{array}\right) \neq 0
$$

by assumption, we can apply Lemma 2.6 again and we deduce $A_{h} \notin E(I)$, which concludes the proof.

q.e.d.

Remark 2. 7. It was questioned by E. Horikawa whether every $E(I)_{m}=\{A \in E(I)|| A \mid=m\}$ can be written as $E(I)_{m}=\left\{A \in Z_{0}^{n}|| A \mid\right.$ $\left.=m, A \geqq A_{0}\right\}$ for some $A_{0}$ if $E(I)_{m} \neq \phi$.

Theorem 2.2 shows that when $n=2$ and $\operatorname{ch} K=0$,

$$
E(I)_{m}=\{(m-j, j) \mid 0 \leqq j<q(m)\} \text { with } q(m)=\operatorname{dim}_{k} I_{m} .
$$

This is nothing but what Horikawa expected.

But when $n=3$ and $\operatorname{ch} K=0$, consider $I=\left(z_{1}^{2}, z_{2}^{2}, z_{1} z_{2}\right)$. We then have $E(I)_{2}=\{(2,0,0),(1,1,0),(0,2,0)\} \not \nexists(1,0,1)$. If $\operatorname{ch} K=p>0$ and $n=2$, consider $l=\left(z_{1}^{p}, z_{2}^{p}\right)$. Then it is easy to see that $E(I)_{p}=\{(p, 0)$, $(0, p)\}$. Moreover, we know in fact that for $\tilde{I}=I K\left[z_{1}, \cdots, z_{n}, z_{n+1}\right]$, where $I$ is a homogeneous ideal in $K\left[z_{1}, \cdots, z_{n}\right]$, we have

$$
E(\tilde{I})=E(I) \times Z_{0}
$$

Thus the answer to Horikawa's question is negative when $\operatorname{ch} K>0$ or $n \geqq 3$, where we exclude the trivial case $n=1$.

\section{References}

[1] Hironaka, H., Idealistic exponents of singularity, in "Algebraic Geometry, The Johns Hopkins Centennial Lectures” (J.-I. Igusa, ed.), Johns Hopkins Univ. Press, 1977, 52-125. 
